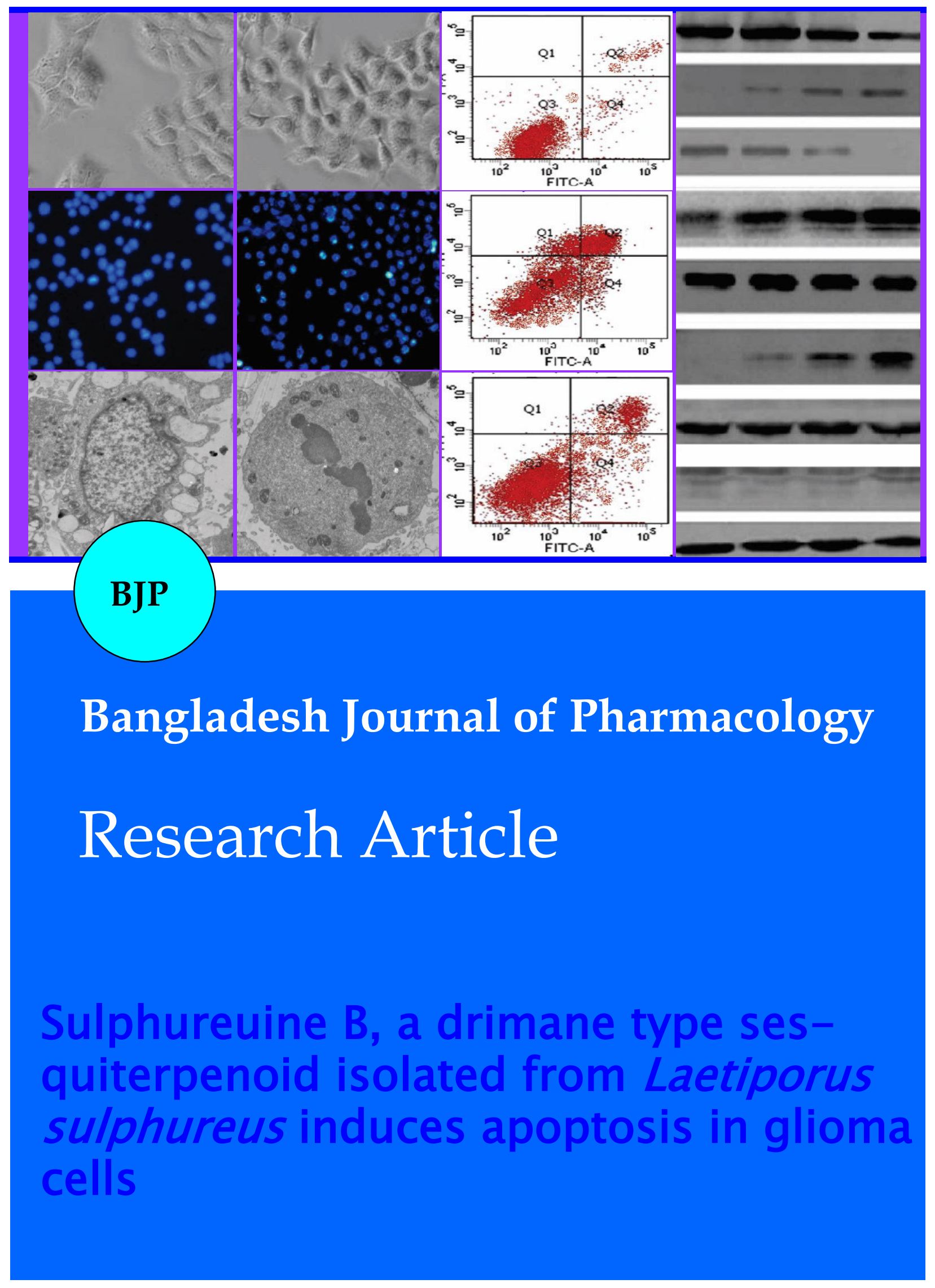




\title{
Sulphureuine B, a drimane type sesquiterpenoid isolated from Laetiporus sulphureus induces apoptosis in glioma cells
}

\author{
Jing-Wei Zhang', Gong-Ling Wen ${ }^{2}$, Lei Zhang', Dong-Mei Duan' ${ }^{3}$ and Zhong-Hai Ren4 \\ ${ }^{1}$ Department of Medical Oncology, Nan Yang City Center Hospital, Henan 437 009, China; ${ }^{2}$ Department of Medical \\ Neurology, Nan Yang City Center Hospital, Henan 437 009, China; ${ }^{3}$ Department of Medical Oncology, Nan Yang Medical \\ College, Henan 473 000, China; ${ }^{4}$ Department of Oncology, Nan Yang City Center Hospital, Henan 437 009, China.
}

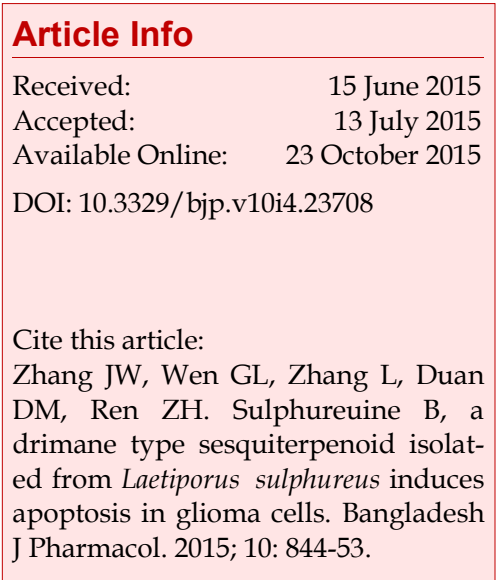

\begin{abstract}
A drimane type sesquiterpenoids, sulphureuine B was isolated from the edible mushroom Laetiporus sulphureus and its antiproliferative properties were investigated using U-87MG glioma cells. It was observed that sulphureuine B-induced apoptosis in U-87MG cells and the mechanisms involved are endoplasmic reticulum stress, mitochondrial and death receptor mediated pathways. Endoplasmic reticulum stress was identified from the results of enormous cytoplasmic vacuolation, CHOP elevation and caspase-12 cleavage. Further, we found that treatment of sulphureuine B-induced PERK, IRE1a, and ATF6a activations. In addition, sulphureuine B-induced Bcl-2 downregulation, cleavage of PARP, and caspase- 8 activation were also affected. All these experimental results clearly revealed that sulphureuine B-induced apoptosis mediated through endoplasmic reticulum stress, mitochondrial, and death receptor signaling pathways.
\end{abstract}

\section{Introduction}

Malignant gliomas are the major life-threatening primary brain tumor with the average survival of less than a year due to the ineffectiveness of radiation and other medical care (Legler et al., 1999). Glioblastomas existing as a spread over to wide area with invasion into typical brain but customarily repeated or developed after radiation as focal point (Garden et al., 1991), indicating that only a small proportion of tumor cells are accountable for regrowth. Even though there occurs the availability of treatment such as combination of surgery, radiation, chemotherapeutics for malicious glioblastomas, still these are considered as locally protruding tumors suffered with poor prediction. A survey from European Organization for Research and Treatment of Cancer (Stupp et al., 2005) displayed that the total viability was increased two more months with associated adjuvant radiation therapy with temozolomide chased by associated temozolomide when compared with the radiation treatment alone. Glioblastomas are very harmful neoplasms which can manifest clear angiogenesis with increased vascular endothelial growth factor expressions which further assist blood vessel production using precursors of endothelium (Plate et al., 1992, Plate et al., 1994). Also it is proved that in the glioblastoma medical care, either surgical treatment followed by radiation treatment or the surgery followed by chemotherapy yields a mean survival of less than 12 months (Fine et al., 1993). Earlier immunotherapy treatments for glioma tumor have concentrated on submissive and non-specific strategies and resulted uncertain benefits (Zeltzer et al., 1999).

Sesquiterpenoids are compounds possessing 15-carbons and can be obtained biosynthetically from 5-carbon isoprene units or can be industrially synthesized using feed stocks of monoterpenoids. These compounds attracted industrial attention due to their beneficial to the characteristic flavors and smell of spices, flowers, 
and herbs (Bauer et al., 1997). Bioactive metabolites are found to be rich in mushroom sources. For example, lanostanoid triterpenes was isolated from Laetiporus sulphureus by Francisco and his group studied apoptosis induction in human myeloid leukemia cells, HL-60 (Francisco et al., 2004). L. sulphureus, is an edible mushroom and it belongs to Polyporaceae family. It is extensively spread in North America, Asia and Russia (Petrović et al., 2013). L. sulphureus is a member of Aphyllophorales fungi class and is very often distributed on conifers and hardwoods. It has been used in Asian folk medicine for long time (Zjawiony, 2004). Mainly, the L. sulphureus higher fungus is familiar for its enormous chemical components which includes triterpenes, sesquiterpenoids, alkaloids, and also well known for its biodiversity (Shiono et al., 2005).

Rapior et al narrated that the L. sulphureus has more or less pleasant odor (Rapior et al., 2000). The wide availability of fungi and their easy attainment make them more popular among the natural products community evaluation program (Berdy, 2005). The concentration towards L. suphureus was brought into attention due to the eburicoic acid biosynthesis in 1960's and there obtained $30 \%$ of L. sulphureus dry weight from this triterpenoid acid (Goad et al., 1966). Recent studies showed that natural products acquired biological activity and it contains enzymes which are specific to catalyze their biosynthesis (Li and Siehr, 1980; Alquini et al., 2004; Kobayashi and Kim, 2003). Recent reports also showed that sesquiterpenoids possessed antitumor activities (Weber et al., 2004; Tipton et al., 2003; El Ashry et al., 2003) but their molecular mechanism towards the actions remain unclear.

The protein folding and its activity were regulated by multifunctional organ called endoplasmic reticulum. This endoplasmic reticulum appeared as a most important site of regulating the cell homeostasis and especially in controlling unfolded protein which is established to play a crucial role in cancer and other contagious diseases. The insufficient glucose contribution may affect the secretory protein pathway glycosylation as well as the production of ATP's. These two factors resulted in the stockpile of unfolded proteins in endoplasmic reticulum and lead to cause endoplasmic reticulum stress (ER stress). The functions of modification in the post translation, accumulation of newly synthesized proteins, folding, and calcium storage regulation was operated and maintained by endoplasmic reticulum (Shoemarker et al., 2005). These are extremely important functions for the survival of cells such that their disorder can lead to cell damage, ER stress and finally apoptosis. Various pathways are activated by ER stress to eradicate the cell damage and these pathways are known as unfolded protein response (UPR). This UPR signal possesses dual role of weakening the damage related with ER stress or upon cell death inducement through apoptosis (Ellgaard and
Helenius, 2003; Wang et al., 2014). ER stress gained more attention towards molecular pathway and this can be modified to produce cytotoxicity in glioma and that can be a feasible aim to introduce new chemotherapeutic drugs to treat glioma cancer (Chakrabarti et al., 2011). However, there is no report mentioning about the mechanism of sulphureuine B-induced cell death in glioma cancer cells and this study was carried out to investigate the antiproliferative action of sulphureuine $\mathrm{B}$ and its mechanism of cell death in U-87 MG glioma cell lines. The comprehensive mechanisms, if explained, can help as to how the sulphureuine B can serve as a powerful drug candidate for future cancer treatment.

\section{Materials and Methods \\ Extraction and isolation of sulphureuine B}

Sulphureuine B (Figure 1) was extracted from the fruiting elements of L. sulphureus. Sulphureuine B was further isolated and purified according to the literature

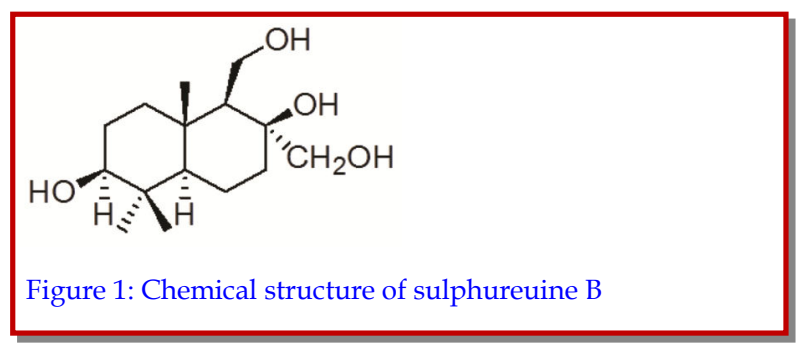

method (He et al., 2015). L. sulphureus fruiting bodies were collected at Fenyi, Jiangxi Province, China. Culture medium contains glucose $(5 \%)$, pork peptone $(0.15 \%)$, yeast $(0.5 \%), \mathrm{KH}_{2} \mathrm{PO}_{4}(0.05 \%)$, and $\mathrm{MgSO}_{4}(0.05$ $\%)$. The initial $\mathrm{pH}$ was adjusted to 6.0 , and then carried out fermentation using Erlenmeyer flask for one week till the mycelium biomass reached to the maximum. Later it was transferred to a fermentation tank $(20 \mathrm{~L})$ at $24^{\circ} \mathrm{C}, 250 \mathrm{rpm}$ for 20 days, and ventilation was set to 1.0 air volume/culture volume/min. The culture broth (20 L) was concentrated under vacuum and extracted with ethylacetate. The organic layer was evaporated in vacuum with crude yield of $5.8 \mathrm{~g}$, which was separated by sephadex LH-20 (methanol) to get its fractions. Later it was subjected to a silica gel column and eluted with petroleum ether : acetone (1:1) to give subfractions (200 $\mathrm{mg}$ ). It was then further purified by sephadex LH-20 (methanol) and silica gel column (petroleum ether: acetone, from 30:1 to 10:1) yield sulphureuine (2.5 mg) as colorless oil. ESI-MS m/z 272 [M]+; HR-EIMS m/z $272.1984[\mathrm{M}]+$ (calcd for $\mathrm{C}_{15} \mathrm{H}_{28} \mathrm{O}_{4}, 272.1988$ ). The structure of sulphureuine $\mathrm{B}$ was identified by the physicochemical methods and spectral data with $98 \%$ purity.

\section{Materials}

The MTT reagent 3-(4,5-dimethylthiazol-2-yl)-2,5-diphenyltetrazolium bromide), DMSO, Hoechst 33258, 
rhodamine 123 and all the solvents and regents used for biological experiments were obtained from SigmaAldrich Company (USA). The antibodies against Bax, Bcl-2, caspases 3, 8, 9 and 12, ATF-6a, FADD, fas, $\beta$ actin, Thr 981 (p-PERK), PARP, Bcl-XL, BID, CHOP, GRP 78, Ser 51 (p-eIF 2a), IRE 1a, XBP 1sand HRP conjugated antibodies were obtained from Santa Cruz Biotechnolgy (USA). Z-VADFMK and ECL were purchased from Cell signaling technology (Cell Signaling Technology, USA).

\section{Cell lines and culture}

U-87MG glioma cells were obtained from American Type Culture Collection (USA). U-87MG cells were cultured using Dulbecco's modified Eagle's medium (GIBCO, USA) supplemented with fetal calf-serum $(10 \%)$, streptomycin $(100 \mu \mathrm{g} / \mathrm{mL})$, penicillin $(100 \mathrm{IU} /$ $\mathrm{mL})$ and L-glutamine $(0.02 \%)$ and maintained at $37^{\circ} \mathrm{C}$ with $5 \% \mathrm{CO}_{2}$ in a humidified atmosphere.

\section{Cytotoxicity}

The MTT assay was carried out to study inhibitory action of sulphureuine B on U-87 MG and its cell viability. The cell lines at a density of $5 \times 10^{3}$ cells/well were seeded on 96 well microplates and kept for incubation for about 24 hours. After one day incubation, these cells were treated with various drug concentrations and their cell growth was measured. Cell viability was expressed as absorbance ratio of treatment to control and their absorbance was recorded at $540 \mathrm{~nm}$ using V-730 UVvisible spectrophotometer (Jasco, USA).

\section{Hoechst and rhodamine staining}

The cells were stained with Hoechst 33258 and/or rhodamine 123 dye. For the Hoechst dye staining, cells were resuspended at 106 cells $/ \mathrm{mL}$ and stained with the dye at a concentration of $5 \mathrm{mg} / \mathrm{mL}$ for $90 \mathrm{~min}$ at $37^{\circ} \mathrm{C}$. After the incubation, cells were kept in serum media at $48^{\circ} \mathrm{C}$ to prohibit leakage of Hoechst dye from the cells. For the rhodamine 123 staining, 106 cells/mL were exposed to $0.1 \mathrm{mg} / \mathrm{mL}$ of rhodamine 123 dye for $20 \mathrm{~min}$ at $37^{\circ} \mathrm{C}$ in the dark. The cells were then washed and resuspended with the serum-enriched media and kept on ice. For combined staining, the same method was used as described for the Hoechst staining, but rhodamine 123 was added during the last 20 min of the incubation. Propidium iodide $(2 \mathrm{mg} / \mathrm{mL})$ was added to the final suspension before the flow cytometric sorting to exclude dead cells from the flow cytometric profile. The fluorescence microscopy was used to determine the cell morphology (Leica Microsystems, Germany).

\section{Dual staining annexin-V/PI assay}

The apoptosis participation in sulphureuine B-induced cell death was investigated using dual staining annexin$\mathrm{V} /$ propidium iodide assay. Cells $\left(1 \times 10^{6}\right)$ were washed and resuspended with PBS. Apoptotic cells were identi- fied by double supravital staining with recombinant FITC (fluorescein isothiocyanate)-conjugated annexin-V and propidium iodide, using the annexin-V-fluos staining kit according to the manufacturer's instructions. Flow cytometric analysis was performed immediately after supravital staining. Data acquisition and analysis were performed in a Becton Dickinson FACSCalibur flow cytometer using CellQuest software. Before sulphureuine B treatment with U-87MG cells, 2 $\mu \mathrm{M}$ of Z-VAD-FMK pan caspase inhibitor was also added.

\section{Mitochondrial membrane potential measurement}

In this experiment, rhodamine 123 , a membrane permeable fluorescent cationic dye was used to determine the measurement of mitochondrial membrane potential. In brief, cells were treated with sulphureuine B and incubated with rhodamine 123 for about $30 \mathrm{~min}$ at $37^{\circ} \mathrm{C}$ in dark. It is then harvested and resuspended in PBS $(200 \mu \mathrm{L})$ and subsequently measured using attune NxT acoustic focusing cytometer (Life Technologies, New York, USA).

\section{Western blot}

The U-87MG cells were first harvested and washed twice using PBS in cold. Later it was lysed in whole cell lysis buffer which are proteinase inhibitors supplied for 1 hour at $4^{\circ} \mathrm{C}$ and centrifuged. After centrifugation, Pierce BCA protein assay kit (Life Technologies, New York, USA) was used to measure the concentration of proteins. The equal content of total protein was separated using 12\% SDS PAGE and then transferred onto the PVDF membrane (Life Technologies, New York, USA). At room temperature, skimmed milk of $5 \%$ was used to block the membranes for about 1 hour and then incubated with primary antibodies overnight at $4^{\circ} \mathrm{C}$ and HRP-conjugated secondary antibody for 2 hours at room temperature and then finally visualized by ECL substrates.

\section{Transmission electron microscope (TEM) studies}

This study was carried out as reported in the previous literature methods (Cheng et al., 2009). U-87MG cells were treated with $50 \mu \mathrm{M}$ sulphureuine $\mathrm{B}$ for the indicated time periods. The collected cells were fixed with $\mathrm{NaCl} /$ Pi containing 3\% glutaraldehyde, postfixed with $\mathrm{NaCl} / \mathrm{Pi}$ containing $1 \% \mathrm{OsO}_{4}$. The samples were dehydrated in graded alcohol, embedded and sectioned. Ultrathin sections were stained with uranyl acetate and lead citrate, and examined using a JEM-1200 transmission electron microscope.

\section{si-RNA transfection}

In this experiment, cells were first transfected with si$\mathrm{CHOP} /$ si-NC at a final concentration of $25 \mathrm{nM}$ using lipofectamine reagent according to the manufacturer's guidelines. The transfected cells were used for subse- 
quent experiments 24 hours later. si-NC (si RNA's) act as a negative control. After 24 hours, the transfected cells were utilized for further experiments.

\section{Statistical analysis}

Statistical analyses were performed using Student's ttest. All the represented data were performed in at least three independent measurements. All the data are expressed as mean $\pm \mathrm{SD}$ and $\mathrm{p}<0.05$ is considered as statistically significant.

\section{Results}

Anti-proliferation and apoptosis inducement against $U$ $-87 M G$ cells

MTT assay was used to determine the cell viability by treating U-87MG cells with various concentrations of sulphureuine B $(5,10,25,50$, and $100 \mu \mathrm{M})$ for 24,36 and 48 hours. Time-dependent and dose-dependent responses were identified from MTT results with an $\mathrm{IC}_{50}$ value of $16 \mu \mathrm{M}$ at 24 hours (Figure 2).

To further investigate the reducing cell survival induced by sulphureuine B, we examined morphological changes in the cells using phase contrast microscopy method. Membrane swelling and apoptosis morphological changes were identified while treating the sulphureuine B when compared to that of control group (Figure 3; upper row). These observations were further tested with Hoechst staining.

The regular distribution of low dense fluorescence was observed in the control whereas condensed, bright fluorescence and fragmentation was observed in the case of sulphureuine B treated cells (Figure 3; middle row). Sulphureuine B-treated U-87MG cells showed the apoptotic characte-ristics such as condensation, vacuolation of cytoplasm and nuclear fragmentation (Figure 3; lower row). All these observations support

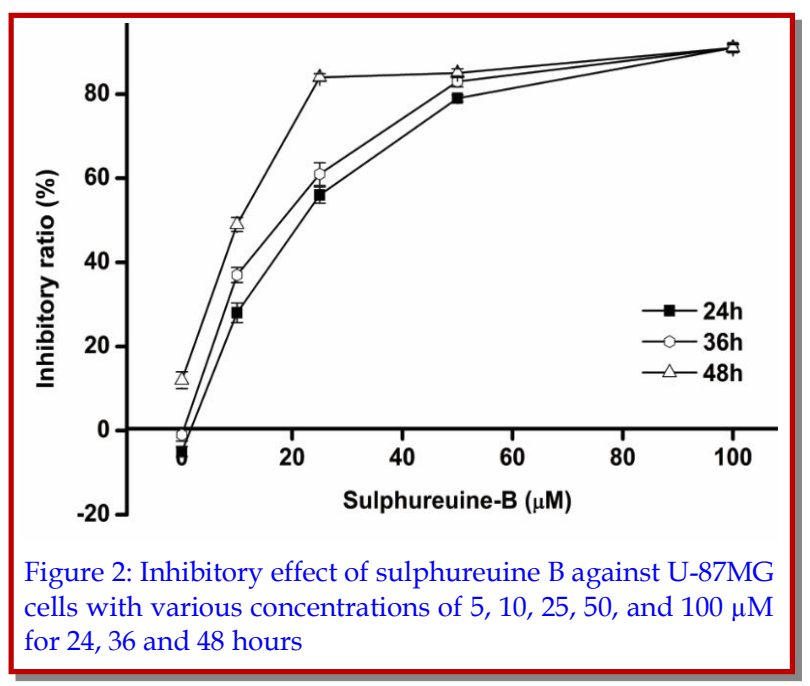

for the apoptosis inducement of sulphureuine B in U87MG cells.

\section{Participation of caspase activation in apoptosis}

Figure 4a showed the PARP cleavage was induced by sulphureuine $\mathrm{B}$, indicating the activation of caspase which is considered as notable characteristics for apoptosis. In addition, we further studied the caspase activation was induced by sulphureuine $B$ and we used western blot analysis to detect the caspases-3, -8 and -9 .

Results depicted in Figure 4A clearly disclosed the procaspase-3, -8 and -9 decrease and significant elevation in the cleaved caspase-3, caspase- 8 and caspase- 9 , suggesting the caspase activation. To find out whether the cell death induced by sulphureuine B was caspase dependent, we pre-treated U-87MG cells with $5 \mu \mathrm{M}$ pan caspase inhibitor, Z-VAD-FMK before treatment with sulphureuine B. Results indicated that treatment of ZVAD-FMK suppressed sulphureuine B induced cell death. We observed the cell death induced by

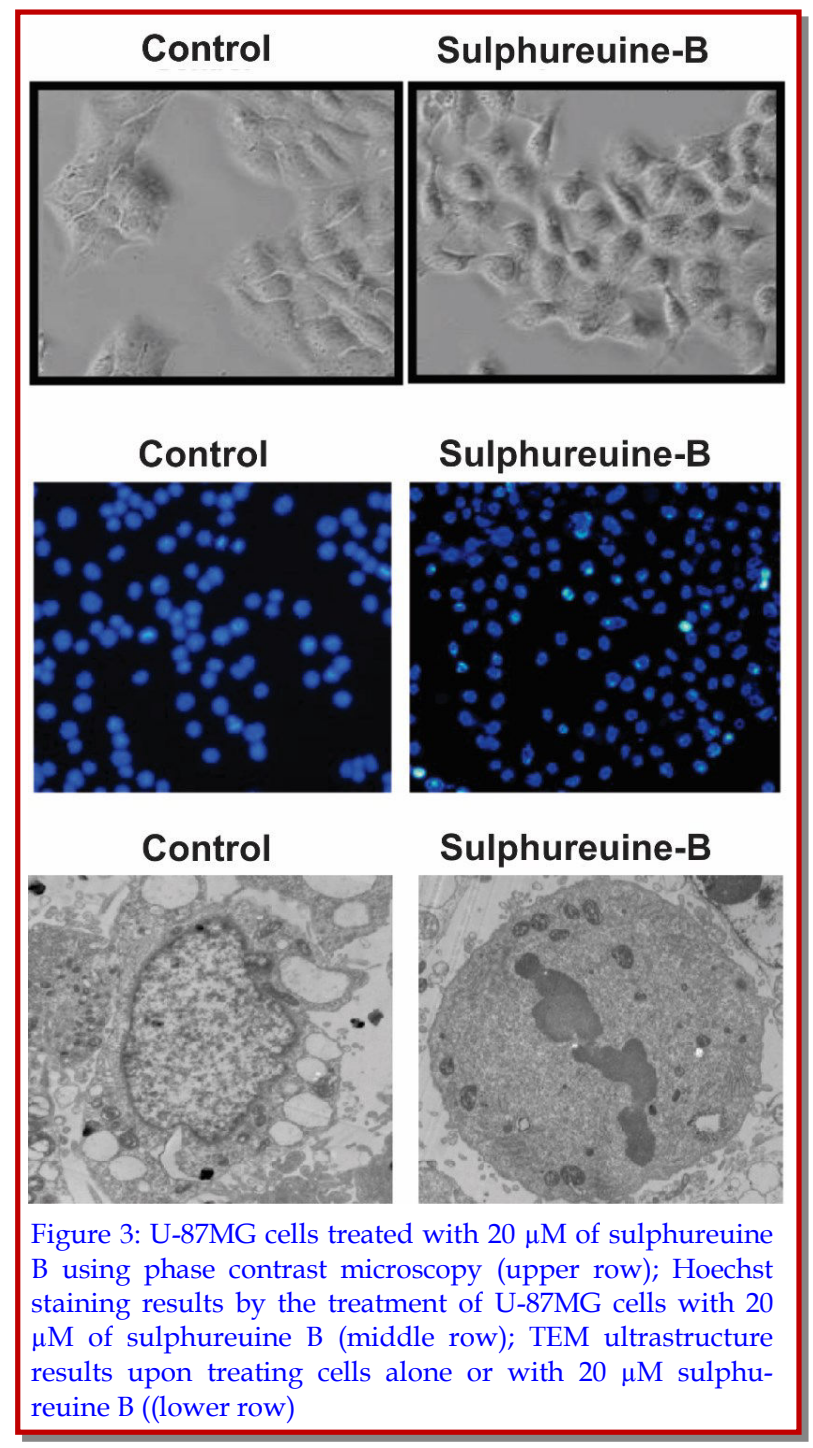




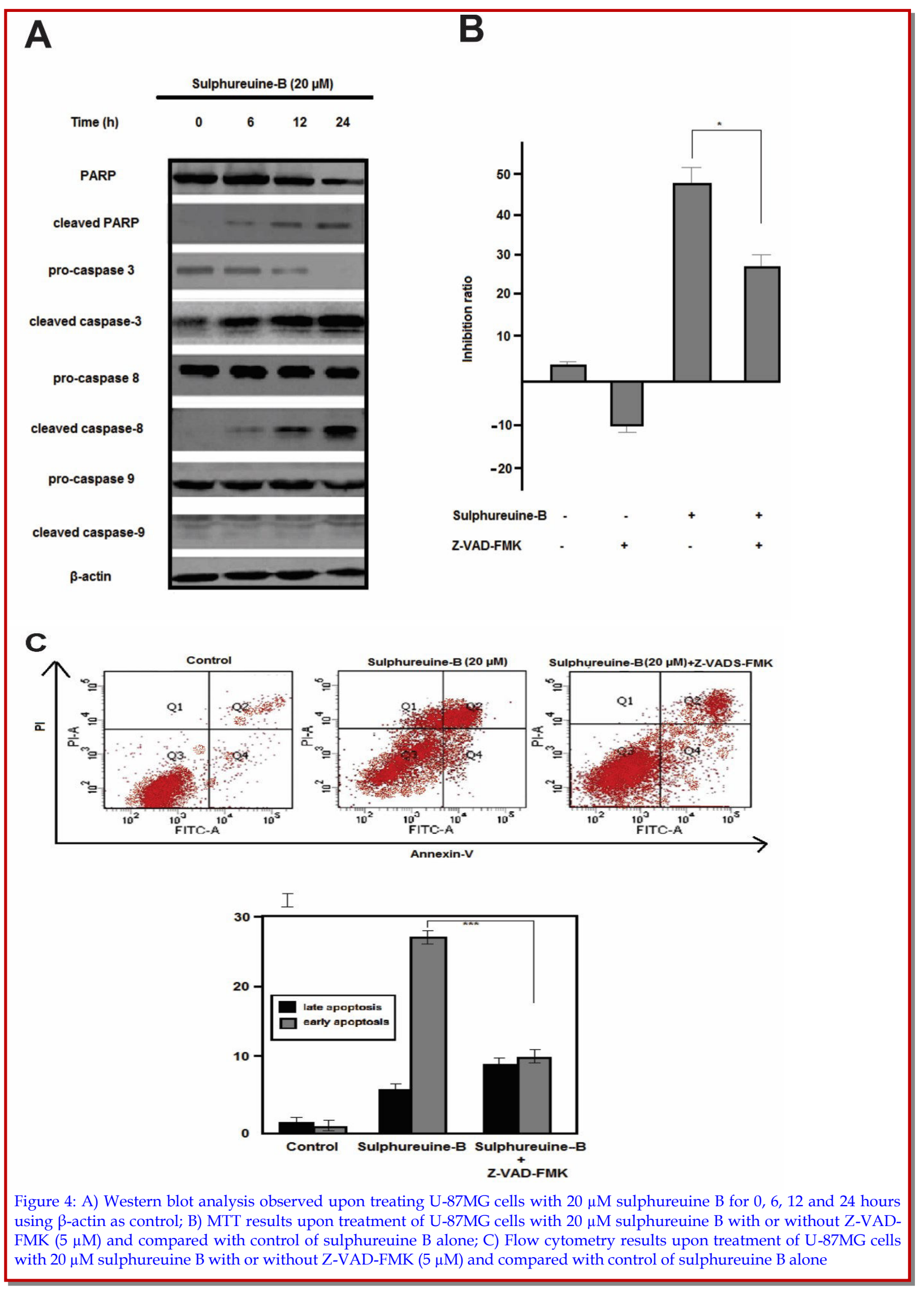


sulphureuine B was reduced from the level of $45.3 \pm$ $0.3 \%$ to $24.6 \pm 2.8$ ) when it underwent with Z-VADFMK co-incubation (Figure 4B). Further, annexin-V/PI flow cytometry results revealed that treatment of $\mathrm{Z}$ VAD-FMK reduced that apoptosis cell population upon sulphureuine B inducement. It was also identified that the late apoptosis ratio was decreased from the level of $26.8 \pm 1.0 \%$ to $9.7 \pm 0.6 \%$ upon $\mathrm{z}$-VAD-FMK cotreatment (Figure 4C). These results clearly indicated the partial involvement of caspase activation in the sulphureuine B induced apoptosis.

\section{Apoptosis in intrinsic and extrinsic pathways}

Caspase- 9 and -8 activations indicated that sulphureuine $B$ induced apoptosis may be proceeded through mitochondrial-mediated intrinsic pathway and death receptor mediated extrinsic pathway. The mitochondrial membrane potential was damaged by the ratio of Bcl-2/Bax protein down regulation. Hence, we performed rhodamine 123 staining to determine the mitochondrial membranes integrity of the cells by using flow cytometry analysis and the fluorescence microscopy. Results showed that treatment of sulphureuine B leads to MMP decrease in a time dependent manner as given in Figure 5A and 5B. Western blot analysis detected many proteins such as Bax, Bcl-2, Bcl-xL of Bcl2 family and fas, FADD of death receptor related proteins. The results indicated that Bax expression was significantly increased whereas $\mathrm{Bcl}-2$ and $\mathrm{Bcl}-\mathrm{xL}$ protein levels are found to be decreased (Figure 5C). Also, Fas \& FADD protein expression levels are also increased. In addition, we found that treatment of sulphurueines B induced the Bid cleavage, which is a caspase 8 downstream protein (Figure 5D). These resulted proved that sulphu-reuine B stimulated apoptosis is mediated through both extrinsic and intrinsic pathways.

\section{Endoplasmic reticulum stress and unfolded protein response}

Enormous cytoplasmic vacuolation was identified in U87MG cells upon treatment with sulphureuine B for about 24 hours (Figure 6A). A normal phenotype was observed with the control group cells. On comparison with control group, sulphureuine B treated cells showed the apoptotic characteristics such as cytoplasmic vacuolation, condensation and nuclear fragmentation. Prolonged treatment with sulphureuine B leads to the formation of hypervacuolation (Figure 6B). Further, the cytoplasmic vacuolation induced by sulphureuine $B$ was not due to autophagy due to the non-inducement of autophagosome formation by sulphureuine B.

It is possible that sulphureuine B induced endoplasmic reticulum changes in morphology may have parallel effect with ER stress and its malfunction. We determined whether the sulphureuine B induces ER stress via the detection of caspase-12, GRP78, and CHOP expression levels. As shown in Figure 6C, results showed that upon treatment with sulphureuine $B$, the caspase-12 activation was observed along with the upregulation of GRP78 and CHOP expression levels in time dependent response. ER stress can cause to happen UPR via the activation of PERK, ATF6, and IRE1 signaling proteins and hence we studied the sulphureuine B effects on 3 pathway signaling of UPR using immune-blotting experiment. The results showed that upon treatment with sulphureuine B, auto-phosphorylation of PERK was identified using Thr981 and also eIF2a phosphorylation was also identified for 6 hours but its corresponding phosphorylated protein level was slightly reduced after 6 hours, which is due to eIF2a dephosphorylation caused by apoptosis elevation. Upon sulphureuine B treatment, ATF6a was found to be readily decreased from 6 hours to 24 hours, evidencing the protein cleavage into the activated form. UPR signal activation was observed from the IRE1a upregulation and XBP1 activation (Figure 6D). Mitochondrial and death receptor signaling pathways are regulated by the pro apoptotic $\mathrm{CHOP}$ protein molecule. In order to examine its involvement in the intrinsic and extrinsic pathways, we studied the caspase- 8 and bcl-2 expressions using siRNA as control and CHOP siRNA transfection studies. Results indicated that there is a decrease in $\mathrm{CHOP}$ by using the particular $\mathrm{CHOP}$ siRNA. Also, CHOP expression decrease further reduced the activation of caspase- 8 and regained the expression of Bcl-2. CHOP inhibition also blocked PARP cleavage induced by sulphureuine B (Figure 6E). All these results clearly revealed the significant importance of CHOP in sulphureuine B-induced apoptosis.

\section{Discussion}

Terpenoids are distributed word wide and it belongs to wide class of secondary metabolites of lipophilic characteristics and derived from iso-pentyl pyrophosphate (Harborne and Toma's-Barbera'n 1991; Harrewijn et al., 2001). The enormous variety of terpenoids exists in plants, and they can produce main constituents of essential oil from the extracts of plants. An early report demonstrated that L. sulphureus extracts contains large amount of hydrocarbons say about approximately $80 \%$ and $43.1 \%$ of acetone \& dichloromethane and methanol extracts, respectively. Among them, nonane, n-decane and 4-methyl nonane were found to be in more percentages. $\mathrm{Wu}$ et al identified 40 main volatile components and quantified (Wu et al., 2005). It is proved that sesquiterpenoids such as a and $\beta$ tumerones, curlone, are the turmeric oil constituents possessing antioxidative and mosquitocidal properties (Jayaprakashaet al., 2002; Roth et al., 1998). Production of E2 and NO prostaglandin was also inhibited utilizing these components (Hong et al., 2002; Lee et al., 2002). In recent years, natural product substrates provides an effective sources for anti-cancer treatment (Pan et al., 


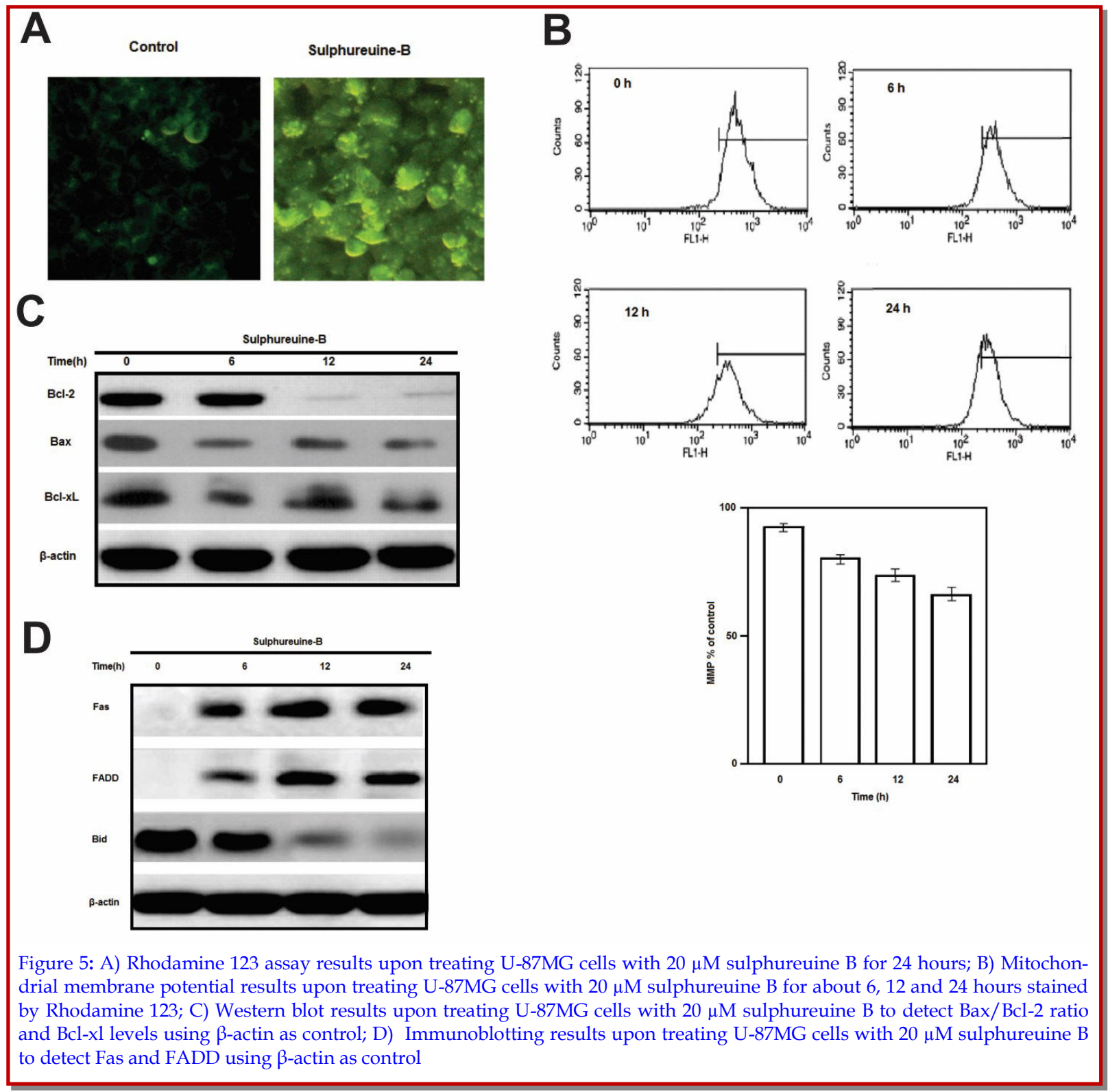

2012). Sulphureuine B was isolated from the natural compound of L. sulphureus. Current study deals with inhibitory effects of sulphureuine B towards the glioma U-87MG cells for the first time as a dose and dependent responses. The sulphureuine B induced apoptosis was also elaborately studied. The mechanism involved in its action was also described as via ER stress, mitochondrial, and death-receptor pathways.

The ER stress in cancer cells involvement is found to be in enormous amount in the anticancer therapies and the reports demonstrated that autophagy and apoptosis in glioma cancer cells was induced by ER stress (Shen et al., 2014). Also, reports showed that apoptosis observed in glioma cells was normally concentrated on ROS generation, cell cycle arrest and the mitochondrial mediated pathway (Chang et al., 2004, Sun et al., 2009, $\mathrm{Lv}$ et al., 2013). From our results, we observed that eIF2a \& PERK phosphorylation, IRE1a up-regulation, ATF6a cleavage, caspase 12 cleavage, CHOP \& GRP78 expression and XBP1s expression was induced by sulphureuine B. All these results evidenced for the sulphureuine B induced apoptosis mediated through endoplasmic reticulum stress.

Earlier reports predicted that mitochondrial and deathreceptor pathways are integrated with endoplasmic reticulum induced apoptosis (Zhu et al., 2011). From the results obtained in our studies, we identified Bcl-2/Bax ratio \& Bcl-xl suppression, caspases-8 \& 9 activation, Bid cleavage, mitochondrial potential membrane loss, Fas \& FADD proteins upstream which are the major 


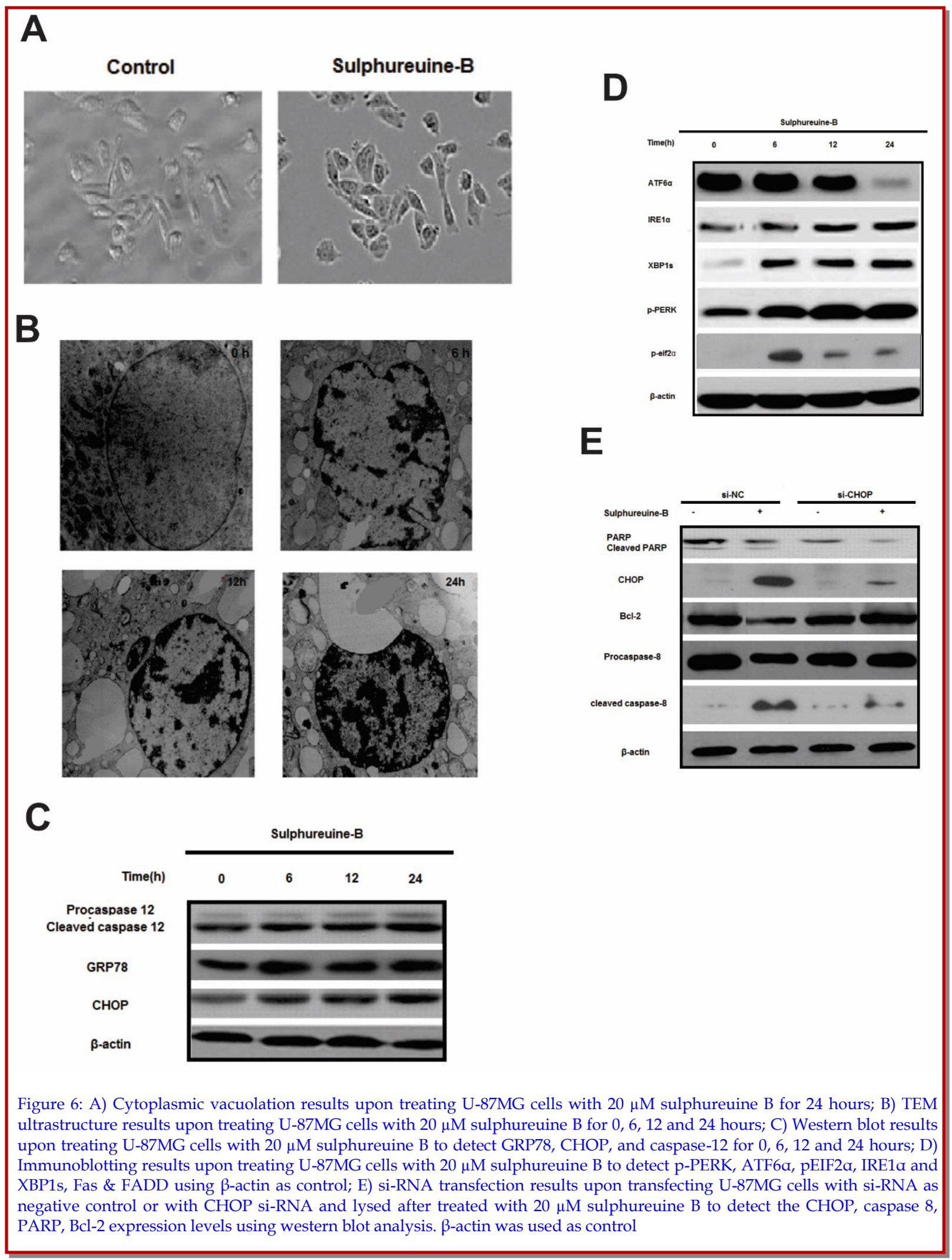


characteristic features for the function of mitochondrial and death-receptor pathways. Si-RNA transfection results came out with $\mathrm{CHOP}$ expressions. Further, it inhibits PARP cleavage and activates caspase- 8 . These results disclosed the vital role of $\mathrm{CHOP}$ in endoplasmic reticulum stress stimulated apoptosis on sulphureuineB treated U-87MG cells.

\section{Conclusion}

Sulphureuine B obtained from L. sulphureus can function as a favorable antiproliferative agent which are potent to inhibit the U-87MG glioma cells operating via endoplasmic reticulum stress, mitochondrial and death receptor signaling pathways. Our findings supports for utilizing sulphureuine B as a powerful anticancer drug for the treatment of malignant glioblastoma.

\section{References}

Alquini G, Carbonero ER, Rosado FR, Cosentino C, Lacomini M. Polysaccharides from the fruit bodies of the basidiomycete Laetiporus sulphureus (Bull. Fr.) Murr. FEMS Microbiol Lett. 2004; 230: 47-52.

Bauer KD, Garbe, Surburg H. Common fragrance and flavor materials: Preparation, properties and uses. 3rd ed. New York, Wiley-VCH, 1997.

Berdy J. Bioactive microbial metabolites: A personal view. J Antibiot. 2005; 58: 1-26.

Chakrabarti A, Chen AW, Varner JD. A review of the mammalian unfolded protein response. Biotechnol Bioeng. 2011; 108: 2777-93.

Chang YC, Chou FP, Huang HP, Hsu JD, Wang CJ. Inhibition of cell cycle progression by penta-acetyl geniposide in rat $\mathrm{C} 6$ glioma cells. Toxicol Appl Pharmacol. 2004; 198: 11-20.

Cheng Y, Qiu F, Ye YC, Guo ZM, Tashiro S, Onodera S, Ikejima T. Autophagy inhibits reactive oxygen species-mediated apoptosis via activating p38- nuclear factor-kappa B survival pathways in oridonin-treated murine fibrosarcoma L929 cells. FEBS J. 2009; 276: 1291-306.

El Ashry ES, Rashed N, Salama OM, Saleh A. Components, therapeutic value and uses of myrrh. Pharmazie 2003; 58: 6368.

Ellgaard L, Helenius A. Quality control in the endoplasmic reticulum. Nat Rev Mol Cell Biol. 2003; 4: 181-91.

Fine HA, Dear KB, Loeffler JS, Black PM, Canellos GP. Metaanalysis of radiation therapy with and without adjuvant chemotherapy for malignant gliomas in adults. Cancer (Phila.). 1993; 71: 2585-97.

Francisco L, Jose' Q, Augusto R, Francisco E, Jaime B. Lanostanoid triterpenes from Laetiporus suphureus and apoptosis induction on HL-60 human myeloid leukemia cells. J Nat Prod. 2004; 67: 2008-11.

Garden AS, Maor MH, Yung WK, Bruner JM, Woo SY, Moser
RP, Lee YY. Outcome and patterns of failure following limited-volume irradiation for malignant astrocytomas. Radiother Oncol. 1991; 20: 99-110.

Goad LJ, Hammam SA, Dennis A. Goodwin TW. Biosynthesis of the phytosterol side chain. Nature 1966; 210: 1322-24.

Harborne JB, Toma's-Barbera'n FA (ed.). Ecological chemistry and biochemistry of plant terpenoids. New York, Oxford University Press, 1991.

Harrewijn P, van Oosten MA, Piron PG. Natural terpenoidsas messengers: A multidisciplinary study of their production, biological functions, and practical applications. Boston, USA, Kluwer Academic Publishers, 2001.

He JB, Tao J, Miao XS, Bu W, Zhang S, Dong ZJ, Li ZH, Feng T, Liu JK. Seven new drimane-type sesquiterpenoids from cultures of fungus Laetiporus sulphureus. Fitoterapia 2015; 102: 1-6.

Hong $\mathrm{CH}$, Noh MS, Lee WY, Lee SK. Inhibitory effects of natural sesquiterpenoids isolated from the rhizome of Curcuma zedoariaon prostaglandin E2 and nitric oxide production. Planta Med. 2002; 68: 545-47.

Jayaprakasha G.K, Jena BS, Negi PS, Sakariah KK. Evaluation of antioxidant activities and antimutagenicity of turmeric oil: A byproduct from curcumin production. $\mathrm{Z}$ Naturforsch. 2002; 57: 828-35.

Kobayashi H, Kim H. Characterization of aspartic proteinase from basidiomycete, Laetiporus sulphureus. Food Sci Technol Res. 2003; 9: 30-34.

Lee SK, Hong CH, Huh SK, Kim SS, Oh OJ, Min HY, Park KK, Chung WY, Hwang JK. Suppressive effect of natural sesquiterpenoids on inducible cyclooxygenase (COX-2) and nitric oxide synthase (iNOS) activity in mouse macrophage cells. J Environ Pathol Toxicol Oncol. 2002; 21: 141-48.

Legler JM, Ries LA, Smith MA, Warren JL, Heineman EF, Kaplan RS, Linet MS. Brain and other central nervous system cancers: Recent trends in incidence and mortality. J Natl Cancer Inst. 1999; 91: 1382-90.

Li SMW, Siehr DJ. Endogenous respiration of Polyporus sulphureus. Mycologia 1980; 72: 64-72.

Lv L, Zheng L, Dong D, Xu L, Yin L, Xu Y, Qi Y, Han X, Peng J. Dioscin, a natural steroid saponin, induces apoptosis and DNA damage through reactive oxygen species: A potential new drug for treatment of glioblastoma multiforme. Food Chem Toxicol. 2013; 59: 657-69.

Pan L, Chai HB, Kinghorn AD. Discovery of new anticancer agents from higher plants. Front Biosci (Schol Ed). 2012; 4: 142-56.

Petrović J, Glamočlija J, Stojković DS, Ćirić A, Nikolić M, Bukvički D, Guerzoni ME, Soković MD. Laetiporus sulphureus, edible mushroom from Serbia: Investigation on volatile compounds, in vitro antimicrobial activity and in situ control of Aspergillus flavus in tomato paste. Food Chem Toxicol. 2013; 59: 297-302.

Plate KH, Breier G, Weich HA, Mennel HD, Risau W. Vascular endothelial growth factor and glioma angiogenesis: Coordinate induction of VEGF receptors, distribution of VEGF protein and possible in vivo regulatory mechanisms. 
Int J Cancer. 1994; 59: 520-29.

Plate KH, Breier G, Weich HA, Risau W. Vascular endothelial growth factor is a potential tumour angiogenesis factor in human gliomas in vivo. Nature 1992; 359: 845-48.

Rapior S, Konska G, Guillot J, Andary C, Bessiere JM. Volatile composition of Laetiporus sulphureus. Cryptogamie Mycol. 2000; 21: 67-72.

Roth GN, Chandra A, Nair MG. Novel bioactivities of Curcuma longa constituents. J Nat Prod. 1998; 61: 542-45.

Shen S, Zhang Y, Wang Z, Liu R, Gong X. Bufalin induces the interplay between apoptosis and autophagy in glioma cells through endoplasmic reticulum stress. Int J Biol Sci. 2014; 10: 212-24.

Shiono Y, Tamesada Y, Muravayev YD, Murayama T, Ikeda M. N-Phenethylhexadecanamide from the edible mushroom Laetiporus sulphureus. Nat Prod Res. 2005; 19: 363-66.

Shoemarker M, Hamilton B, Dairkee SH, Cohen I, Campbell MJ. In vitro anticancer activity of twelve Chinese medicinal herbs. Phytother Res. 2005; 19: 649-51.

Stupp R, Mason WP, van den Bent MJ, Weller M, Fisher B, Taphoorn MJ, Belanger K, Brandes AA, Marosi C, Bogdahn U, Curschmann J, Janzer RC, Ludwin SK, Gorlia $\mathrm{T}$, Allgeier A, Lacombe D, Cairncross JG, Eisenhauer E, Mirimanoff RO. European Organisation for Research and Treatment of Cancer Brain Tumor and Radiotherapy Groups; National Cancer Institute of Canada Clinical Trials Group: Radiotherapy plus concomitant and adjuvant temozolomide for glioblastoma. N Engl J Med. 2005; 352: 987 $-96$.
Sun JY, Yang H, Miao S, Li JP, Wang SW, Zhu MZ, Xie YH, Wang JB, Liu Z, Yang Q. Suppressive effects of swainsonine on $\mathrm{C} 6$ glioma cell in vitro and in vivo. Phytomedicine 2009; 16: 1070-74.

Tipton DA, Lyle B, Babich H, Dabbous MKH. In vitro cytotoxic and anti-inflammatory effects of myrrh oil on human gingival fibroblasts and epithelial cells. Toxicol. In Vitro. 2003; 17: 301-10.

Wang WA, Groenendyk J, Michalak M. Endoplasmic reticulum stress associated responses in cancer. Biochim Biophys Acta. 1843; 2014: 2143-49.

Weber RWS, Mucci A, Davoli P. Laetiporic acid, a new polyene pigment from the wood-rotting basidiomycete Laetiporus sulphureus (Polyporales, fungi). Tetrahedron Lett. 2004; 45: $1075-78$

Wu S, Zorn H, Krings U, Berger R. Characteristic volatiles from young and aged fruiting bodies of wild Polyporus sulfureus (Bull.: Fr.). Fr J Agric Food Chem. 2005; 53: 4524-28.

Zeltzer PM, Moilanen B, Yu JS, Black KL. Immunotherapy of malignant brain tumors in children and adults. Child's Nerv Syst. 1999; 15: 514-28.

Zhu GY, Li YW, Tse AK, Hau DK, Leung CH, Yu ZL, Fong WF. 20(S)-Protopanaxadiol, a metabolite of ginsenosides, induced cell apoptosis through endoplasmic reticulum stress in human hepatocarcinoma HepG2 cells. Eur J Pharmacol. 2011; 668: 88-98.

Zjawiony JK. Biologically active compounds from Aphyllophorales (Polypore) fungi. J Nat Prod. 2004; 67: 300-10.

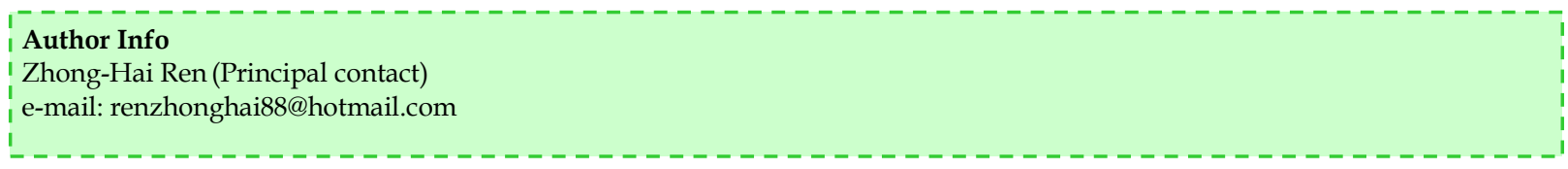

